\section{Clozapin und Quetiapin schneiden am besten ab}

Unter Risperidon wurde mehr Anti-EPSMedikation gebraucht als unter Clozapin, Olanzapin, Quetiapin und Ziprasidon, unter Ziprasidon mehr Anti-EPS-Medikation als unter Olanzapin und Quetiapin und unter Zotepin wiederum mehr als unter Clozapin. Zwischen Amisulprid und Olanzapin, Risperidon sowie Ziprasidon wurden keine statistisch signifikanten Unterschiede festgestellt. Unter Quetiapin wurde signifikant weniger Anti-EPSMedikation als unter Olanzapin, Risperidon und Ziprasidon benötigt.

Kommentar: Antipsychotika der zweiten Generation (SGA) zeigen Unterschiede in ihrer Potenz, EPS zu induzieren. Die Unterschiede waren zwar gering, dennoch sollte bei der Auswahl eines Antipsychotikums auf diese Unterschiede geachtet werden. Eine Einschränkung der Aussagekraft dieser Metaanalyse ergibt sich aus der mangelnden Be- rücksichtigung unterschiedlicher Dosen, was aber nicht den Autoren, sondern vielmehr den eingeschlossenen Studien, die in der Regel keinen breiten Dosisbereich untersuchten, anzulasten ist.

Prof. Dr. med. Hans-Peter Volz

Rummel-Kluge et al. Second-generation antipsychotic drugs and extrapyramidal side-effects: a systematic review and meta-analysis of head-to-head comparisons. Schizophrenia Bulletin 2012; 38: 167-77

\title{
Hohe Dopaminsynthese deutet auf drohenden Ausbruch einer Psychose hin
}

Die vorliegende Studie konnte beim Vergleich von gesunden Kontrollpersonen mit Menschen mit einem hohen Psychoserisiko zeigen, dass der Ausbruch einer Psychose mit einer vermehrten präsynaptischen striatalen Dopaminsynthese verknüpft ist.

$\mathrm{D}$ opamin wird seit Jahrzehnten eng mit der Ätiopathogenese psychotischer Störungen verknüpft, vor allem mit einer Fehlsteuerung der präsynaptischen dopaminergen Aktivität. Speziell wurde hier eine dopaminerge Überfunktion im Striatum, die bereits vor Beginn der klinisch feststellbaren psychotischen Symptomatik einsetzt, postuliert. In dieser Phase der „Krankheitsentstehung “", der prodromalen Phase, werden häufig eine Verminderung sozialer Funktionen und diskrete psychotische Symptome gesehen. Siehe hierzu auch das klassische Konzept der Basisstörungen.

\section{Noch nicht Erkrankte mit hohem Psychoserisiko}

Die Arbeitsgruppe wandte sich dieser Problematik zu, indem sie Menschen mit einem sehr hohen Risiko für die Entstehung eine Psychose untersuchte - allerdings ohne, dass diese bei den Personen bereits ausgebrochen war. Anschließend verfolgte sie diese Gruppe im Längsschnitt weiter. Im Laufe dieser „Follow-Up“-Periode entwickelte nun tatsächlich ein Teil dieser Teilnehmer eine Psychose. Diese Entwicklung ermöglichte einen retrospektiven Vergleich zwischen „Ultra-high-risk“-Teilnehmern, die eine Psychose entwickelten, und den Teilnehmern, die keine Psychose entwickelten.
Die 30 „Ultra-high-risk“-Teilnehmer (Comprehensive Assessment of At-Risk Mental Status-Kriterien für ultra-high risk

\section{Anzeige}

für Psychosen) wurden zweimal untersucht: Initial und nach einer mindestens dreijährigen Beobachtungsphase. Als Vergleichsgruppe dienten 29 gesunde Kon- trollpersonen. Verwandt wurde ein PET mit markiertem DOPA. DOPA wird in der Präsynapse verwandt, um Dopamin zu synthetisieren, eine hohe Aktivität deutet auf eine vermehrte Dopaminsyntheseleistung.

\section{Psychopathologie und Dopamin-Synthesekapazität}

Die Patienten, die von einem Ultra-highrisk-Stadium in eine Psychose übergingen („Transition-group“) zeigten einen höhere Dopamin-Synthese-Kapazität im Striatum als die Vergleichsgruppe Gesunder. Zudem zeigte sich eine positive Korrelation zwischen der Dopamin-SyntheseKapazität und der Psychopathologie. Die "Transition-group" zeigte auch eine höhere Dopamin-Synthese-Kapazität als die „Non-transition-group“, also jene Menschen, die zwar einen ultra-high-riskStatus zeigten, aber dann keine Psychose entwickelten.

Kommentar: Diese Ergebnisse zeigen, dass der Ausbruch einer Psychose mit einer vermehrten präsynaptischen striatalen Dopamin-Synthese verknüpft ist. Auch scheint dies ein Prädiktor für den späteren Ausbruch einer psychotischen Störung zu sein.

Prof. Dr. med. Hans-Peter Volz

Howes et al. Dopamine synthesis capacity before onset of psychosis. A prospective [18F]-DOPA PET imaging study. Am J Psychiatry $2011 ; 168: 1311-17$ 